\title{
Polarization of III-nitride blue and ultraviolet light-emitting diodes
}

\author{
J. Shakya, K. Knabe, K. H. Kim, J. Li, J. Y. Lin, and H. X. Jianga) \\ Department of Physics, Kansas State University, Manhattan, Kansas 66506-2601
}

(Received 1 November 2004; accepted 12 January 2005; published online 25 February 2005)

\begin{abstract}
Polarization-resolved electroluminescence studies of III-nitride blue and ultraviolet (UV) light-emitting diodes (LEDs) were performed. The LEDs were fabricated on nitride materials grown by metalorganic chemical vapor deposition on sapphire substrates (0001). Transverse electric (TE) polarization dominates in the InGaN/GaN quantum-well (QW) blue LEDs $\left(\lambda^{\prime}=458 \mathrm{~nm}\right)$, whereas transverse magnetic (TM) polarization is dominant in the AlInGaN QW UV LEDs $(\lambda=333 \mathrm{~nm})$. For the case of edge emission in blue LEDs, a ratio $\left(r=I_{\perp} / I_{\|}\right)$of about 1.8:1 was observed between the EL intensities with polarization $\boldsymbol{E} \perp \boldsymbol{c}$ (TE mode) and $\boldsymbol{E} \| \boldsymbol{c}$ (TM mode), which corresponds to a degree of polarization $\sim 0.29$. The UV LEDs exhibit a ratio $r$ of about $1: 2.3$, corresponding to a degree of polarization $\sim 0.4$. This is due to the fact that the degree of polarization of the bandedge emission of the $\mathrm{Al}_{x} \mathrm{In}_{y} \mathrm{Ga}_{1-x-y} \mathrm{~N}$ active layer changes with $\mathrm{Al}$ concentration. The low emission efficiency of nitride UV LEDs is partly related to this polarization property. Possible consequences and ways to enhance UV emitter performances related to this unique polarization property are discussed. (C) 2005 American Institute of Physics. [DOI: 10.1063/1.1875751]
\end{abstract}

The recent progresses in high-brightness light-emitting diodes (LEDs) based on III-nitride wide band gap semiconductors place LEDs as strong candidates for solid-state lighting technology. Due to their low power consumption, ${ }^{1}$ compact size, and long lifetime as compared to conventional lamps, these LEDs could advantageously replace conventional incandescent as well as florescent lamps for general lighting. In addition, visible LEDs with high external efficiency are currently in high demand for a variety of applications including flat panel displays, printers, and optical interconnects in computers. High efficiency ultraviolet (UV) emitters are particularly sought for applications including chemical and biological agent detection and medical uses. High intensity and high speed UV LEDs could also be used as transceivers for covert nonline-of-sight optical communications.

Currently, UV LEDs with ternary AlGaN or quaternary AlInGaN alloys as active layers have lower external quantum efficiency (QE) than that of blue LEDs with InGaN as an active layer. This discrepancy is partly due to the lower electrical and optical qualities of AlGaN alloys. However, the inherent property of polarization selective emission from these alloys also accounts for the lower QE of UV LEDs. Ill-nitride materials have a wurtzite structure and are anisotropic along and perpendicular to the direction of the $c$ axis. This anisotropy results in various phenomena including the presence of a spontaneous electric polarization, the splitting of the valence bands, and polarized spontaneous emission. ${ }^{2,3}$ The crystal field influences differently on the valence band splittings in GaN and AlN. ${ }^{4}$ Our recent studies revealed the difference between the band structures and fundamental optical transitions in $\mathrm{AlN}$ and GaN., ${ }^{4,5}$ For the materials grown along the $c$ axis, the optical emission involving electrons from the conduction band to the holes in the topmost valence band is polarized along the direction of $\boldsymbol{E} \perp \boldsymbol{c}(\boldsymbol{E} \| \boldsymbol{c})$ in $\mathrm{GaN}$ $(\mathrm{AlN}){ }^{4,5}$ The relative emission intensity of the preferred po-

\footnotetext{
a) Author to whom correspondence should be addressed; electronic mail: jiang@phys.ksu.edu
}

larization (or the degree of polarization) of the emitted light in $\mathrm{Al}_{x} \mathrm{Ga}_{1-x} \mathrm{~N}$ alloys changes continuously with the $\mathrm{Al}$ concentration $(x)$. This preferential emission property has a direct consequence on the emitter performance. As the InGaN QW blue LEDs are predominantly $\boldsymbol{E} \perp \boldsymbol{c}$ polarized, ${ }^{3}$ nitride UV LEDs using AlGaN (or InAlGaN) with a relatively high $\mathrm{Al}$ content as active layers are expected to be predominantly $\boldsymbol{E} \| \boldsymbol{c}$ polarized. $^{5}$

In this letter, we report the polarization resolved studies of light emission from UV and blue LEDs. The III-nitride materials used were grown by metalorganic chemical vapor deposition on sapphire (0001) substrates. Figures 1(a) and 1(b) show the schematic diagrams of our $458 \mathrm{~nm}$ blue and $333 \mathrm{~nm}$ UV LED structures, respectively. The sources used were trimethylgallium for $\mathrm{Ga}$, trimethylaluminum for $\mathrm{Al}$, trimethylindium for In, and ammonia for nitrogen. For $\mathrm{Mg}$ doping, bis-cyclopentadienyl-magnesium was transported into the growth chamber during growth while $\mathrm{SiH}_{4}$ was used for Si doping. The active region for the blue LEDs was eight periods of $\operatorname{~n}_{0.2} \mathrm{Ga}_{0.8} \mathrm{~N} / \mathrm{GaN}$ multiple quantum well (MQW) while for UV LEDs this was an $\mathrm{Al}_{0.11} \mathrm{In}_{0.03} \mathrm{Ga}_{0.86} \mathrm{~N} /$

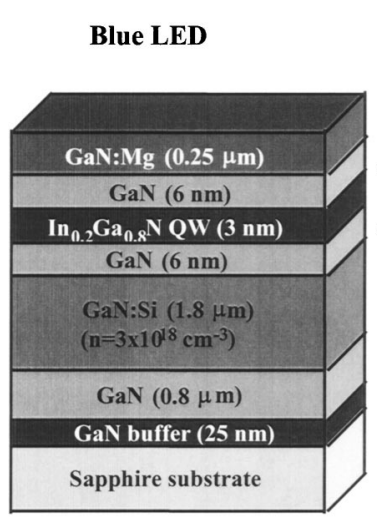

(a)
UV LED

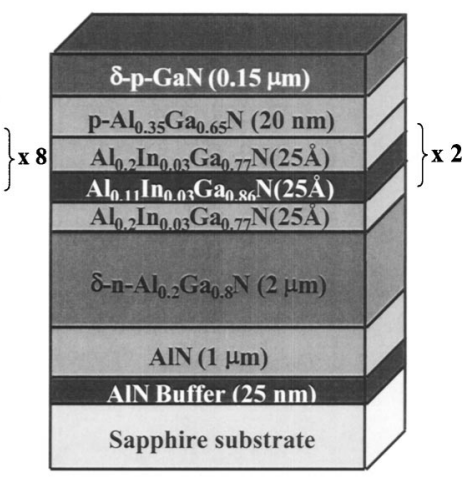

(b)
FIG. 1. Schematic diagrams of (a) $458 \mathrm{~nm}$ blue and (b) $333 \mathrm{~nm}$ UV LED structures. 

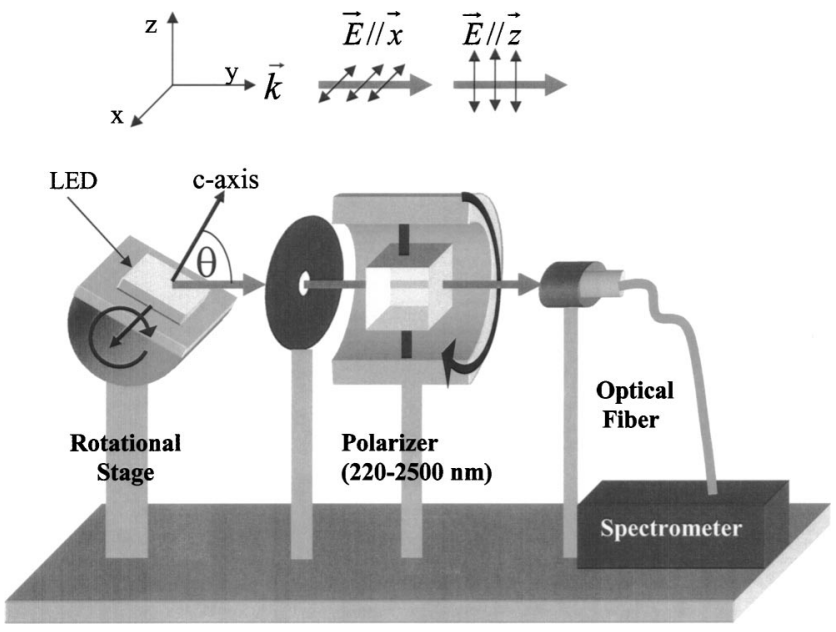

FIG. 2. Schematic diagram of the experimental setup to measure the angle and polarization resolved EL spectra of LEDs. The rotational stage has an $X-Y-Z$ translator stage holding LEDs and can be rotated about the $x$ axis. Light emitted along the $y$ axis passing through a pinhole and cube polarizer is ultimately detected by a spectrometer. The polarizer cube was mounted on $360^{\circ}$ rotational stage which selects either the $\boldsymbol{E} \| \boldsymbol{x}$ or the $\boldsymbol{E} \| \boldsymbol{z}$ component.

$\mathrm{Al}_{0.2} \mathrm{In}_{0.03} \mathrm{Ga}_{0.77} \mathrm{~N}$ double quantum well. Hexagonal mesa LEDs of side length $120 \mu \mathrm{m}$ were fabricated as previously described. ${ }^{6}$

Figure 2 shows the schematic diagram of the experimental setup to measure the angle and polarization resolved electroluminescence (EL) spectra from LEDs. The rotational stage has an $X-Y-Z$ translator stage holding LEDs and can be rotated about the $x$ axis. Light emitted along the $y$ axis passing through a pinhole and cube polarizer is coupled to UV/ visible optical fiber and ultimately detected by a spectrometer (Ocean Optics PC2000). The polarizer can be rotated about the $y$ axis to resolve $\boldsymbol{E} \| \boldsymbol{x}$ (electric field along $x$ axis) and $\boldsymbol{E} \| \boldsymbol{z}$ (electric field along $z$ axis) polarizations. $\theta$ is the angle between $c$ axis of nitride materials and the light detection direction ( $y$ axis). For $\theta=90^{\circ}$, edge emitted EL is collected and the $\boldsymbol{E} \| \boldsymbol{x}$ and $\boldsymbol{E} \| \boldsymbol{z}$ components represent light polarized perpendicular $(\boldsymbol{E} \perp \boldsymbol{c})$ and parallel $(\boldsymbol{E} \| \boldsymbol{c})$ to the $c$ axis and are identified as TE and TM modes, respectively. For $\theta=0^{\circ}$, considering no anisotropy in the plane perpendicular with $\boldsymbol{c}$, both $\boldsymbol{E} \| \boldsymbol{x}$ and $\boldsymbol{E} \| \boldsymbol{z}$, polarized emission intensities are expected to be identical. For $\theta \neq 90^{\circ}$, the measured $\boldsymbol{E} \| \boldsymbol{x}$ and $\boldsymbol{E} \| \boldsymbol{z}$ components are not sole TE and TM modes of LED emission.

Figures 3(a) and 3(b) show the polarization resolved EL spectra of blue and UV LEDs respectively, with $\theta=75^{\circ}$. Both LEDs were driven with a $20 \mathrm{~mA}$ forward current. $\boldsymbol{E} \| \boldsymbol{x}$ polarization dominates in the blue LED, whereas $\boldsymbol{E} \| \boldsymbol{z}$ polarization is dominant in the UV LED. The EL spectra of the blue (UV) LED have a peak intensity at $\lambda=458 \mathrm{~nm}(\lambda$ $=333 \mathrm{~nm}$ ). Unlike some photoluminescence $(\mathrm{PL})$ studies of $\mathrm{GaN},{ }^{7,8}$ no shift in the peak position of the EL spectra was observed between the two polarization components in both LEDs, including the case of $\theta=90^{\circ}$. The low temperature PL studies of $\mathrm{Al}_{x} \mathrm{Ga}_{1-x} \mathrm{~N}$ alloys $(0 \leqslant x \leqslant 1)^{5}$ and GaN/AlGaN $\mathrm{MQW}^{2}$ also revealed the absence of the PL spectral peak position shift between $\boldsymbol{E} \perp \boldsymbol{c}$ and $\boldsymbol{E} \| \boldsymbol{c}$ components, which was attributed to the fact that the selection rule is partially relaxed by the strain, substrate misorientation, and the involvement of the exciton transition. ${ }^{5}$ volvement of the exciton transition.
Downloaded 12 Jul 2010 to 129.118 .86 .59 . Redistribution subject to AlP license or copyright; see http

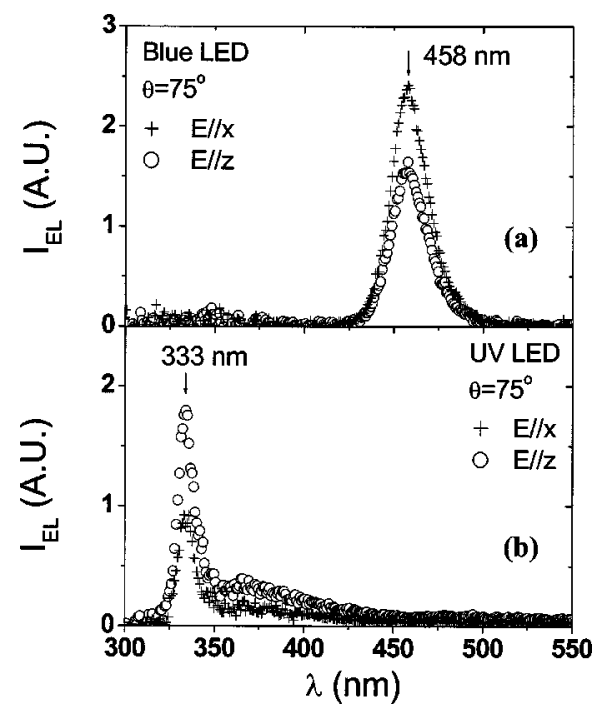

FIG. 3. $\boldsymbol{E} \| \boldsymbol{x}$ and $\boldsymbol{E} \| \boldsymbol{z}$ components of EL spectra of (a) blue LED and (b) UV LED at $\theta=75^{\circ}$. Both LEDs were driven with a $20 \mathrm{~mA}$ forward current.

The angular dependence of the polarization resolved peak EL intensities of blue and UV LEDs is shown in Figs. 4(a) and 4(b), respectively. For $\theta=0^{\circ}$, both the $\boldsymbol{E} \| \boldsymbol{x}$ and the $\boldsymbol{E} \| \boldsymbol{z}$ polarized intensities are equal, which suggests that there is no anisotropy in the growth plane, as expected. Also as expected, the EL intensities decrease as $\theta$ increases. The $\boldsymbol{E} \| \boldsymbol{z}$ component decreases faster than the $\boldsymbol{E} \| \boldsymbol{x}$ component in blue LED, whereas the opposite is true for UV LED. The degree of polarization $P$ is defined by

$$
P=\frac{I_{E \| x}-I_{E \| z}}{I_{E \| x}+I_{E \| z}}
$$

where $P=1(P=-1)$ for completely $\boldsymbol{E} \| \boldsymbol{x}(\boldsymbol{E} \| \boldsymbol{z})$ polarized light. Figure 4(b) shows the degree of polarization of blue and UV LEDs at different angle $\theta$. For blue LEDs the degree of polarization increases from $P=0$ at $\theta=0^{\circ}$ to $P=0.29$ at $\theta=90^{\circ}$ (edge emission). This corresponds to a ratio $(r$

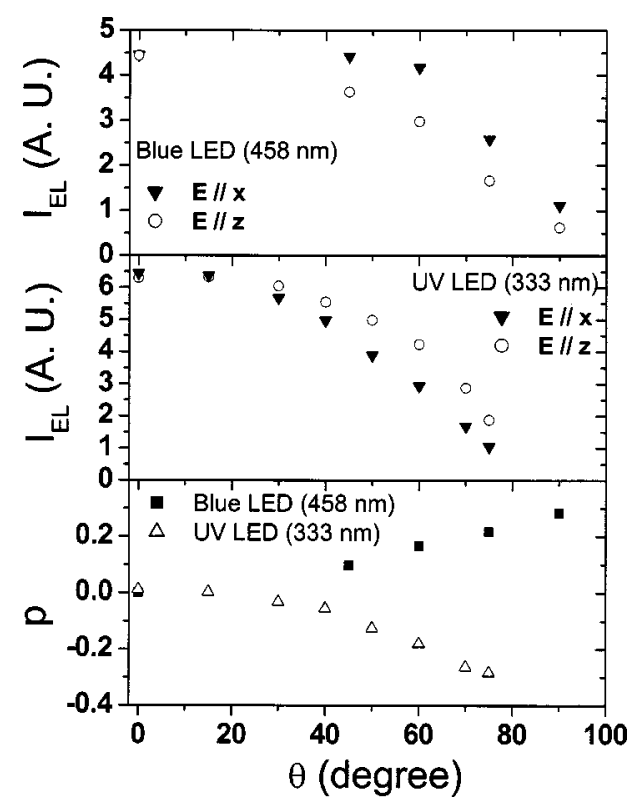

FIG. 4. Angular dependence of the peak EL intensities of $\boldsymbol{E} \| \boldsymbol{x}$ and $\boldsymbol{E} \| \boldsymbol{z}$ components of (a) blue LED and (b) UV LED emissions. (c) The degree of 
$\left.=I_{\perp} / I_{\|}\right)$of about 1.8:1 between the edge emitted EL intensities with polarization $E \perp c$ and $E \| c$. Much higher values of $P$ have been obtained for GaN from low temperature PL studies $(P=0.8 \text { at } 10 \mathrm{~K})^{5}$ and GaN/AlGaN MQW $(P=0.9$ at $5 \mathrm{~K}){ }^{2}$ However, $P$ was found experimentally as well as theoretically to be 0.5 for $\mathrm{GaN}$ from room temperature $\mathrm{PL}$ studies. ${ }^{7}$ In addition to the bulk optical property, the compressive strain favors TE mode, whereas tensile strain favors TM mode in QW structures. ${ }^{9}$ A mixed QW and quantum dot (QD) behavior has also been suggested to be responsible for the reduced $E \perp c$ to $E \|_{c}$ component ratio for blue LEDs. ${ }^{3}$ The smaller $P$ value of our blue LED, as compared to 3:1 $(P=0.5)$ reported in Ref. 3 could be related to strain relaxation and/or mixed QW QD behavior.

As shown in Fig. 4(b), the degree of polarization for UV LEDs decreases from $P=0$ at $\theta=0^{\circ}$ to $P=-0.28$ at $\theta=75^{\circ}$. At $\theta=90^{\circ}$ (edge emitting), the value of $P$ is around -0.4 , which corresponds to a ratio, $r$, of about 1:2.3 between the edge emitted EL intensities with polarization $E \perp c$ and $E \| c$. This reversal in the sign of $P$ cannot be explained by the strain alone, as there is no tensile strain present in both structures. Rather it is expected from the unique band structure due to change in $\mathrm{Al}$ concentration in $\mathrm{Al}_{x} \mathrm{In}_{y} \mathrm{Ga}_{1-x-y} \mathrm{~N}$ from $x=0$ to $x=0.11$. As both $\mathrm{GaN}$ and $\mathrm{InGaN}$ have dominant emission with $E \perp c$ polarization, $\mathrm{Al}$ concentration in the active layer is mainly responsible for the effect of sign reversal of $P$. Though the conduction bands of both AlN and GaN have a $\Gamma_{7}$ symmetry, crystal field splits the valence band differently for the two materials, changing the symmetry of the topmost valence band from $\Gamma_{9}$ in $\mathrm{GaN}$ to $\Gamma_{7}$ in AlN. The consequence therefore is that the light emitted from $\mathrm{GaN}$ (AlN) due to the recombination of the conduction band electrons to the holes at the topmost valence band has a $E \perp c$ $(E \| c)$ polarization. ${ }^{5}$ Edge emitted PL study of $\mathrm{Al}_{x} \mathrm{Ga}_{1-x} \mathrm{~N}$ epilayers shows that $P$ changes continuously from positive values to negative values with increasing $x{ }^{5}$ Compared to the $10 \mathrm{~K}$ PL results of $P=0$ at $x=0.25$ for the edge emission, ${ }^{5}$ we found a more profound polarization reversal effect in the $300 \mathrm{~K}$ EL spectra from an actual UV LED using AlInGaN as an active layer. This might be due to the fact that the layer structure of the UV LED is more complex in comparison to the AlGaN epilayer. Nevertheless, the attribution of the change in polarization to the $\mathrm{Al}$ concentration is consistent with the PL results ${ }^{5}$ as well as the theoretical calculation of the band structure of AlGaN. ${ }^{4}$

As $E \|_{c}$ being the dominant emission in AlGaN-based UV emitters, the light generated in the QW is directed, guided and trapped in the QW plane, most of which is lost due to parasitic absorption in the material. For $\mathrm{AlGaN} / \mathrm{GaN}$ QW UV LEDs $(\lambda=350 \mathrm{~nm})$ with a chip area of $300 \mu \mathrm{m}$ $\times 300 \mu \mathrm{m}$, light generated at the center and guided in the QW plane will be attenuated before it gets out from the edge by a factor of $\mathrm{e}^{-\alpha x} \sim 0.2$, where $\alpha=106 \mathrm{~cm}^{-1}$ is the optical loss in QW. ${ }^{10}$ Currently, for deep UV LEDs $(\lambda<300 \mathrm{~nm})$, the loss is even bigger due to a relatively poor material qual- ity. One of the important issues in deep UV LEDs is devising effective mechanisms to get the light out as soon as it is generated. We have employed interconnected microdisk LED architecture $^{11}$ and two-dimensional (2D) photonic crystals $(\mathrm{PCs})^{12-15}$ to enhance the extraction of light from LEDs. As a larger fraction of light is guided in UV LEDs than in blue LEDs due to this unique polarization property, the enhancement in the power output by improving the extraction of the guided light is more significant in UV LEDs. This explains the discrepancies between the optical power enhancement factors in the 2D PCs incorporated $460 \mathrm{~nm}$ blue LEDs $(=1.63)^{13}$ and $330 \mathrm{~nm} \mathrm{UV} \mathrm{LEDs}(=2.5) .{ }^{14}$ An enhancement factor of 2.9 for PCs incorporated deep UV LEDs $(\lambda$ $=299 \mathrm{~nm})^{16}$ further suggests that the shorter the wavelength the bigger fraction of light is trapped and lost within the devices.

In summary, we have performed angle and polarization resolved EL studies of III-nitride blue and UV LEDs. In contrast to blue LEDs (460 nm), UV LEDs were found to emit predominantly TM polarized light. The degree of polarization $P$ for edge emission of InGaN QW blue LED is 0.29 , which corresponds to a ratio of 1.8:1 for $E \perp c$ (TE mode) and $E \|_{c}$ (TM mode) components. For AlInGaN QW UV LED $(333 \mathrm{~nm})$, the value of $P$ was found to be -0.4 and the corresponding ratio was $1: 2.3$. This emission property makes nitride UV emitters unique and significantly impacts the performance of UV emitters.

The authors wish to acknowledge support by grants from ARO, DOE, NSF, DARPA.

\footnotetext{
${ }^{1}$ S. Nakamura and G. Fasol, The Blue Laser Diode (Springer, Berlin, 1997).

${ }^{2}$ B. Rau, P. Waltereit, O. Brandt, M. Ramsteiner, K. H. Ploog, J. Puls, and F. Henneberger, Appl. Phys. Lett. 77, 3343 (2000).

${ }^{3}$ D. I. Floresscu, D. S. Lee, S. M. Ting, J. C. Ramer, and E. A. Armour, J. Electron. Mater. 32, 1330 (2003).

${ }^{4}$ J. Li, K. B. Nam, M. L. Nakarmi, J. Li, J. Y. Lin, H. X. Jiang, P. Carrier, and S. H. Wei, Appl. Phys. Lett. 83, 5163 (2003).

${ }^{5}$ K. B. Nam, J. Li, M. L. Nakarmi, J. Y. Lin, and H. X. Jiang, Appl. Phys. Lett. 84, 5264 (2004).

${ }^{6}$ J. Shakya, K. H. Kim, J. Y. Lin, and H. X. Jiang, Appl. Phys. Lett. 85, 142 (2004).

${ }^{7}$ K. Domen, K. Horino, A. Kuramata, and T. Tanahashi, Appl. Phys. Lett. 71, 1996 (1997).

${ }^{8}$ D. C. Reynolds, D. C. Look, B. Jogai, A. W. Saxler, S. S. Park, and J. Y. Hahn, Appl. Phys. Lett. 77, 2879 (2000).

${ }^{9}$ W. J. Fan, M. F. Li, and T. C. Chong, J. Appl. Phys. 80, 3471 (1996).

${ }^{10}$ J. Shakya, J. Y. Lin, and H. X. Jiang, Appl. Phys. Lett. 84, 1832 (2004).

${ }^{11}$ S. X. Jin, J. Li, J. Y. Lin, and H. X. Jiang, Appl. Phys. Lett. 77, 3236 (2000).

${ }^{12}$ T. N. Oder, J. Shakya, J. Y. Lin, and H. X. Jiang, Appl. Phys. Lett. 83, 1231 (2003).

${ }^{13}$ T. N. Oder, K. H. Kim, J. Y. Lin, and H. X. Jiang, Appl. Phys. Lett. 84, 466 (2004).

${ }^{14}$ J. Shakya, K. H. Kim, J. Y. Lin, and H. X. Jiang, Appl. Phys. Lett. 85, 142 (2004).

${ }^{15}$ J. Shakya, K. H. Kim, J. Li, J. Y. Lin, and H. X. Jiang, Mater. Res. Soc. Symp. Proc. 798, 163 (2003).

${ }^{16}$ J. Shakya, K. H. Kim, T. N. Oder, J. Y. Lin, and H. X. Jiang, SPIE Proceedings, 2004 Fourth International Conference on Solid-State Lighting (submitted).
} 